\title{
Monte Carlo modelling of NLO DGLAP QCD Evolution in the fully unintegrated form
}

\author{
A. Kusina*i \\ H. Niewodniczański Institute of Nuclear Physics, Polish Academy of Sciences, \\ ul. Radzikowskiego 152, 31-342 Krakow, Poland \\ E-mail: Aleksander.Kusina@ifj.edu.pl \\ S. Jadach \\ H. Niewodniczański Institute of Nuclear Physics, Polish Academy of Sciences, \\ ul. Radzikowskiego 152, 31-342 Krakow, Poland \\ E-mail: Stanislaw.Jadach@ifj.edu.pl
}

\section{Skrzypek}

H. Niewodniczański Institute of Nuclear Physics, Polish Academy of Sciences, ul. Radzikowskiego 152, 31-342 Krakow, Poland

E-mail: Maciej.Skrzypek@ifj.edu.pl

\section{Slawinska}

H. Niewodniczański Institute of Nuclear Physics, Polish Academy of Sciences, ul. Radzikowskiego 152, 31-342 Krakow, Poland

E-mail: Magdalena.Slawinska@ifj.edu.pl

Presently available perturbative QCD calculations combining hard process matrix element with the Parton Shower Monte Carlo programs feature hard process matrix element calculated often beyond the leading order (LO), that is including complete next-to-leading-order (NLO), or even next-to-next-to-leading-order (NNLO) corrections, while Parton Shower is only at the leading order (LO). We report here on a work in progress which demonstrate feasibility of constructing Parton Shower Monte Carlo (PSMC) featuring complete NLO corrections to QCD evolution with respect to the logarithm of the factorization scale. This effort presently covers non-singlet subset of Feynman diagrams contributing to the above QCD evolution. It should be stressed that our approach to the NLO QCD evolution is exclusive, that is giving insight into the fully unintegrated phase space. However, at the inclusive level our implementation agrees exactly with the standard inclusive picture of the NLO DGLAP evolution. Our new approach (after including NLO singlet diagrams) provides a complete method of combining the resummed and fixed order perturbative QCD calculations beyond the LO in a form suitable for the MC implementation, an alternative to the existing ones. First practical applications will include Monte Carlo generators for W/Z production processes in hadron-hadron colliders and lepton-hadron colliders.

35th International Conference of High Energy Physics

July 22-28, 2010

Paris, France

\footnotetext{
*Speaker.

$\dagger$ This work is partly supported by the EU Framework Programme grants MRTN-CT-2006-035505 and by the Polish
} 
As the Large Hadron Collider (LHC) just started to operate, experiments will need high accuracy QCD calculations not only for inclusive observables, but also for multiparton final states, especially in a form of events from Parton Shower Monte Carlo (PSMC) programs. Huge range of energy scales in the PSMCs will require including beyond the LO calculations not only in the hard process part but also in the middle of the shower (ladder). Why 25 years old traditional PSMCs, see refs. [1], are still restricted to the LO level only? Main reasons are that: (A) principal role of PSMCs was to hadronize quark and gluons into hadrons, (B) hadron collider data were until recently rather poor, (C) conceptual problem on the side of the factorization theorems of QCD [2], badly suited for the MC implementation, as they (i) violate 4-momentum conservation, (ii) irreversibly integrate over real emissions (iii) feature huge over-subtractions and cancellations (iv) operate with non-positive distributions, (D) last not least computers were until recently to slow to consider unintegrated NLO corrections inside the PSMC ladder. With the presented works an era of NLO level PSMCs finally comes soon! Possible profits/gains from NLO PSMC are the following: (a) new simpler and cleaner schemes of matching of the hard process ME at NLO and NNLO level with NLO PSMC, (b) natural extensions towards BFKL/CCFM evolution at low $x$, (c) better modelling of low scale phenomena, $Q<10 \mathrm{GeV}$, (d) new MC tools for porting information on parton distributions from one process to another (from DIS at HERA to W/Z production at LHC) and from one scale to another - an alternative for traditional inclusive PDFs. In our work presented here, we take scheme of Curci, Furmanski, Petronzio (CFP) [4] as a guide and we concentrate on the non-singlet NLO evolution for the initial state radiation (ISR) of multiple gluons. NLO corrections are added to each LO kernel in the middle of LO ladder of the PSMC, see below,

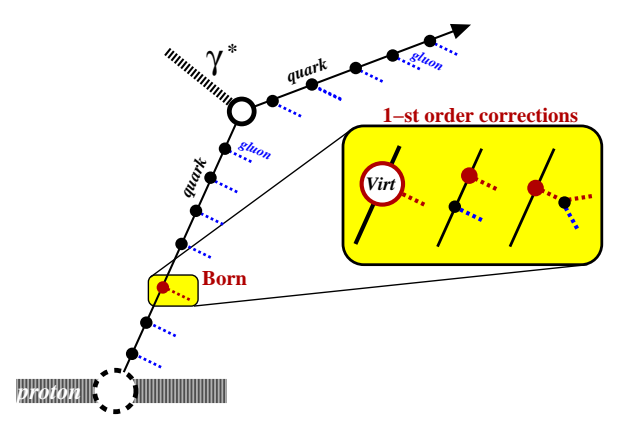

such that at the inclusive level we reproduce NLO DGLAP [3] evolution. As depicted above, our starting point is the LO ISR single ladder like in DIS. Let us start with including NLO corrections to the gluon emission vertex at the top of this LO ladder,

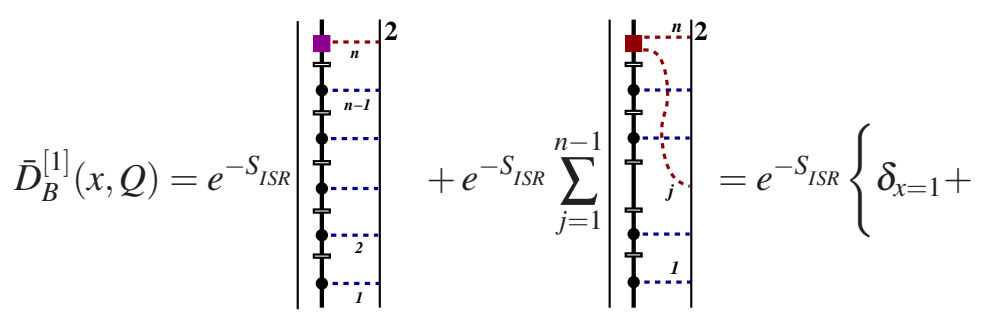

$$
\begin{aligned}
& \left.+\sum_{n=1}^{\infty}\left(\prod_{i=1}^{n} \int_{Q>a_{i}>a_{i-1}} \frac{d^{3} k_{i}}{k_{i}^{0}} \rho_{1 B}^{(1)}\left(k_{i}\right)\right)\left[\beta_{0}^{(1)}\left(z_{n}\right)+\sum_{j=1}^{n-1} W\left(\tilde{k}_{n}, \tilde{k}_{j}\right)\right] \delta_{x=\prod_{j=1}^{n} x_{j}}\right\},
\end{aligned}
$$

Ministry of Science and Higher Education grants No. 1289/B/H03/2009/37 and 153/6.PR UE/2007/7. 
where the Monte Carlo weights reweighting LO distributions to NLO level is given by:

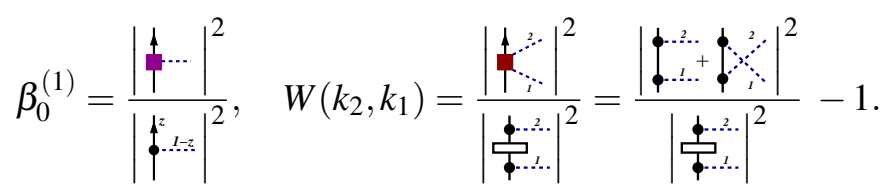

Let us explain first all parts and the ingredients in Eq. (1). First term (ladder) is just the LO ladder multiplied by NLO virtual correction factor for the last vertex, where $\rho_{1 B}^{(0)}\left(k_{i}\right)=\frac{2 C_{F}^{2} \alpha_{s}}{\pi} \frac{1}{k_{i}^{T}} \frac{1+z^{2}}{2}$ is LO Altarelli-Parisi evolution kernel, $\theta_{Q>a_{i}>a_{i-1}}$ indicates the ordering in angle $\left(a_{i}\right.$ is the rapidity related variable defined as $a_{i}=\left|\mathbf{k}_{i \perp}\right| / \alpha_{i}, \alpha_{i}$ is plus variable in Sudakov parametrization), $\frac{d^{3} k_{i}}{k_{i}^{0}}$ is the Lorentz invariant phase-space and $e^{-S_{I S R}}$ is the Sudakov formfactor. The NLO virtual correction

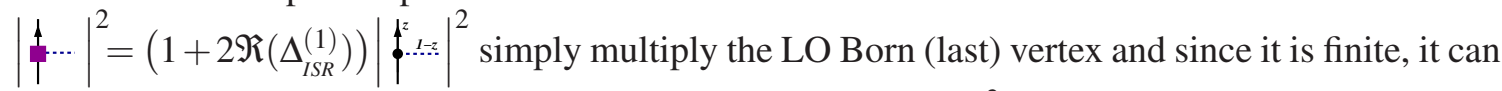
be easily implemented by reweighting the $\mathrm{LO}$ distribution. The $C_{F}^{2}$ real corrections coming from the

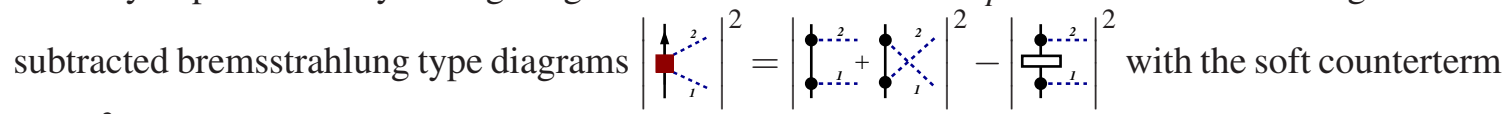
$\left|\frac{1}{\phi_{1}^{2} \ldots}\right|^{2}$ subtracting the LO component affect pairs of partons (act in two particle phase-space) ${ }^{1}$. The distinctive feature of Eq. (1) is the presence of the summation over trailing gluons along the ladder. Without this summation (taking only $W\left(k_{n}, k_{n-1}\right)$ ) we wouldn't have the full NLO correction.

Including the full NLO correction all over the ladder requires to sum over the positions of the real NLO insertions (corrections) in the ladder and adding virtual corrections to all vertices:

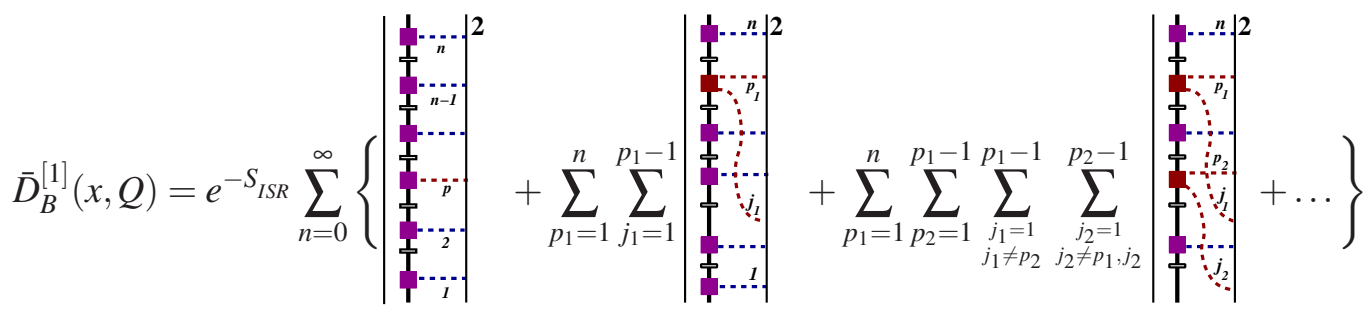

$$
\begin{aligned}
& =e^{-S_{I S R}}\left\{\delta_{x=1}+\sum_{n=1}^{\infty}\left(\prod_{i=1}^{n} \int_{Q>a_{i}>a_{i-1}} d^{3} \eta_{i} \rho_{1 B}^{(1)}\left(k_{i}\right) \beta_{0}^{(1)}\left(z_{p}\right)\right)\left[1+\sum_{p=1}^{n} \sum_{j=1}^{p-1} W\left(\tilde{k}_{p}, \tilde{k}_{j}\right)+\right.\right. \\
& \left.\left.+\sum_{p_{1}=1}^{n} \sum_{p_{2}=1}^{p_{1}-1} \sum_{\substack{j_{1}=1 \\
j_{1} \neq p_{2}}}^{p_{1}-1} \sum_{\substack{j_{2}=1 \\
j_{2} \neq p_{1}, j_{2}}}^{p_{2}-1} W\left(\tilde{k}_{p_{1}}, \tilde{k}_{j_{1}}\right) W\left(\tilde{k}_{p_{2}}, \tilde{k}_{j_{2}}\right)+\ldots\right] \delta_{x=\prod_{j=1}^{n} x_{j}}\right\}
\end{aligned}
$$

The above scheme is tested numerically with 3-digit precision using prototype MC program:
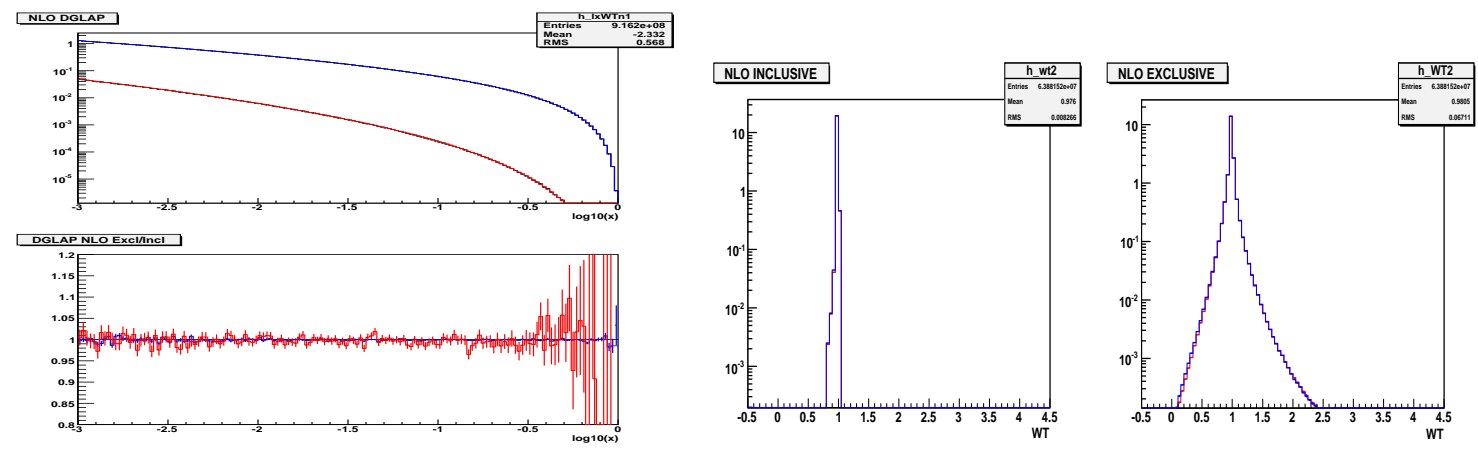

\footnotetext{
${ }^{1}$ In our graphical notation the amplitude square means cut diagrams for both Feynman graph and the counterterm.
} 
Perfect agreement of the new scheme and traditional inclusive approach is seen, especially in the left lower plot, where the ratios for two sums in Eq. (3) are plotted separately. In the right plot we see the weight distributions for inclusive and exclusive approach. The exclusive weight is positive and narrow.

In NLO corrections proportional to $C_{A} C_{F}$ color factor, we get pair production diagram $|b|^{2}$. First question is why we treat this type of corrections in a special way not just include them in the same manner as before. The problem is that it induces Sudakov double logarithm $+S_{F S R}$ (additional singularity when produced pair is collinear) which would ruin the MC weight, if we would restrict ourself to strict NLO only. Resummation/exponentiation for this contribution is mandatory, and new LO ladder on the external leg has to be implemented in the MC - each emitted gluon will cascade into gluon emission ladder. The FSR counterterm $|\cdot \cdot-\cdot|^{2}$ is constructed, encapsulating exactly soft and collinear singularities of $C_{A} C_{F}$ diagrams (both double and single logs). The expression for the $\mathrm{MC}$ distribution with the last emission in the ladder upgraded from the LO to NLO level reads:

$$
\begin{aligned}
& \bar{D}_{N S}^{[1]}(x, Q)=
\end{aligned}
$$

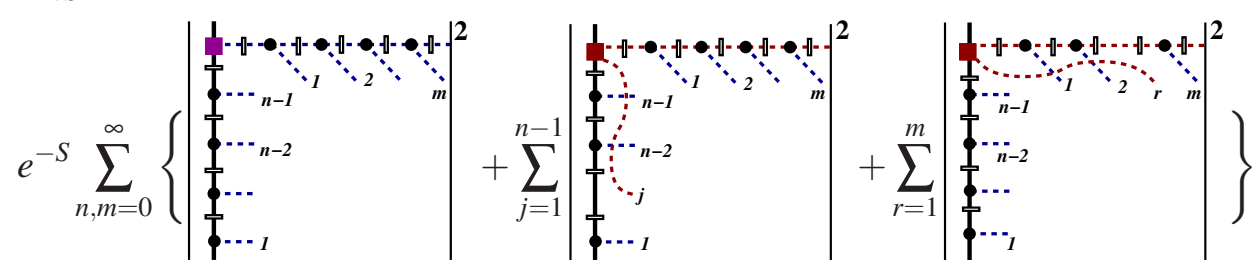

$$
\begin{aligned}
& =e^{-S_{I S R}}\left\{\delta_{x=1}+\sum_{n=1}^{\infty}\left(\prod_{i=1}^{n} \int_{Q>a_{i}>a_{i-1}} d^{3} \eta_{i} \rho_{1 B}^{(1)}\left(k_{i}\right)\right) e^{-S_{F S R}} \sum_{m=0}^{\infty}\left(\prod_{j=1}^{m} \int_{Q>a_{n j}>a_{n(l-1)}} d^{3} \eta_{j}^{\prime} \rho_{1 V}^{(1)}\left(k_{j}^{\prime}\right)\right)\right. \\
& \left.\times\left[\beta_{0}^{(1)}\left(z_{n}\right)+\sum_{j=1}^{n-1} W\left(\tilde{k}_{n}, \tilde{k}_{j}\right)+\sum_{r=1}^{m} W\left(\tilde{k}_{n}, \tilde{k}_{r}^{\prime}\right)\right] \delta_{x=\prod_{j=1}^{n} x_{j}}\right\},
\end{aligned}
$$

where the $\mathrm{MC}$ weights are given by

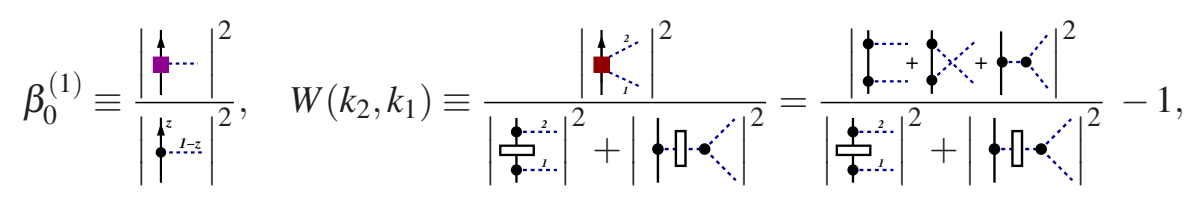

are finite, since all collinear and soft, ISR and FSR, singularities are subtracted. In particular, in the virtual correction $\left|\frac{1}{\uparrow} \cdots\right|^{2}=\left(1+2 \Re\left(\Delta_{I S R}+V_{F S R}-S_{F S R}\right)\right)||^{z}-1-\left.z\right|^{2}$ FSR Sudakov $S_{F S R}$ is subtracted.

Three-digit precision numerical test of the above FSR methodology was done for a single NLO ISR+FSR insertion for $n=1,2$ ISR gluons and infinite number of FSR gluons. 


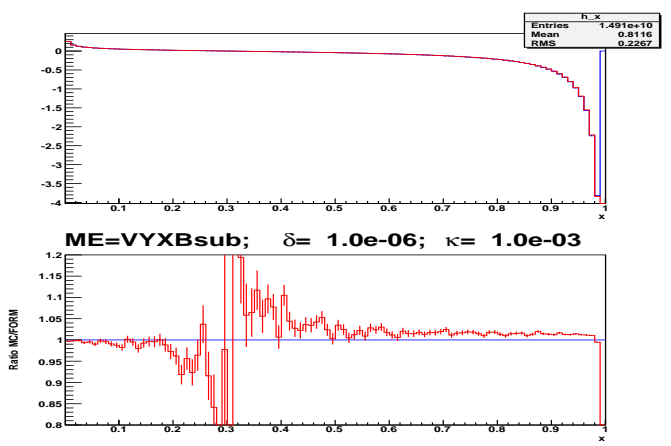

The comparison is done between the MC and analytical results.

The exclusive approach presented here has been tested against an independent MC implementing standard inclusive DGLAP evolution in the NLO level. We can see the perfect agreement of both approaches on the level of few promiles, see [5, 6, 7]. Let us stress that in our exclusive MC scenario we have complete insight into the transverse degrees of freedom up to NLO level, something that is unavailable in other common inclusive or semi-inclusive approaches. Our exclusive NLO MC features good behaviour of the MC weights - weights are positive and narrowly distributed. The techniques presented above are promising and will be exploited in the construction of NLO MC generators for W/Z production @LHC and DIS @HERA. Works related to this ongoing project are reported in refs. $[5,7,8,9,10]$.

\section{References}

[1] T. Sjostrand, Phys. Lett. B157 (1985) 321; B.R. Webber, Nucl. Phys. B238 (1984) 492.

[2] R.K. Ellis et al., Nucl. Phys. B152 (1979) 285; J.C. Collins, D.E. Soper and G. Sterman, Nucl. Phys. B250 (1985) 199; G.T. Bodwin, Phys. Rev. D31 (1985) 2616.

[3] L.N. Lipatov, Sov. J. Nucl. Phys. 20 (1975) 95; V.N. Gribov and L.N. Lipatov, Sov. J. Nucl. Phys. 15 (1972) 438; G. Altarelli and G. Parisi, Nucl. Phys. 126 (1977) 298; Yu. L. Dokshitzer, Sov. Phys. JETP 46 (1977) 64.

[4] G. Curci, W. Furmanski and R. Petronzio, Nucl. Phys. B175 (1980) 27.

[5] S. Jadach, A. Kusina, M. Skrzypek and M. Slawinska, arXiv:1007.2437 [hep-ph].

[6] M. Skrzypek and S. Jadach, PoS E PS-HEP2009, 297 (2009) [arXiv:0909.5588 [hep-ph]].

[7] S. Jadach and M. Skrzypek, Acta Phys. Polon. B 40, 2071 (2009) [arXiv:0905.1399 [hep-ph]].

[8] S. Jadach, M. Skrzypek, A. Kusina and M. Slawinska, PoS RADCOR2009, 069 (2010) [arXiv:1002.0010 [hep-ph]].

[9] A. Kusina, S. Jadach, M. Skrzypek and M. Slawinska, Acta Phys. Polon. B 41, 1683 (2010) [arXiv:1004.4131 [hep-ph]].

[10] M. Slawinska and A. Kusina, Acta Phys. Polon. B 40, 2097 (2009) [arXiv:0905.1403 [hep-ph]]. 